\title{
Resistance to viral infection of super p53 mice
}

\author{
Cesar Mun̄oz-Fontela ${ }^{1}$, Maria Angel Garcia², Isabel Garcia-Cao³, Manuel Collado², \\ Javier Arroyo ${ }^{1}$, Mariano Esteban'2, Manuel Serrano ${ }^{3}$ and Carmen Rivas*,1
}

${ }^{1}$ Departamento de Microbiologia II, Facultad de Farmacia, Universidad Complutense de Madrid, Plaza Ramon y Cajal sn, Madrid 28040, Spain; ${ }^{2}$ Centro Nacional de Biotecnologia, CSIC, Campus Universidad Autonoma de Madrid, Madrid, Spain; ${ }^{3}$ Spanish National Cancer Center (CNIO), Madrid, Spain

\begin{abstract}
Induction of expression of the tumor suppressor p53 after interferon treatment has been recently demonstrated (Takaoka et al., 2003), suggesting an antiviral activity of the protein. In addition, a direct correlation between p53 levels and tumor resistance has been addressed by generating mice with an extra copy of p53 ('super p53' mice) (Garcia-Cao et al., 2002). Here, we show that vesicular stomatitis virus replication in mouse embryo fibroblasts derived from 'super p53' mice is impaired as a result of apoptosis induction via p53 activation. These findings unequivocally demonstrate an antiviral activity of p53, a process that may contribute to inhibit the spread of the virus in vivo.
\end{abstract}

Oncogene (2005) 24, 3059-3062. doi:10.1038/sj.onc.1208477 Published online 14 February 2005

Keywords: p53; vesicular stomatitis virus; vaccinia virus; tumor suppressor; antiviral activity; 'super p53' mice

Interferons (IFNs) are cytokines responsible for conferring an antiviral state on cells and with a role in rejection of malignancies (reviewed by Clemens, 2003). These effects are mediated by the gene products activated or induced following the interaction of IFNs with their cognate receptors(s), involved in a variety of processes, including inflammation, cellular stressresponse programmes and apoptosis (reviewed by Samuel, 2001).

p53 acts as a tumor suppressor through its capacity to induce cell cycle arrest and apoptosis in response to a variety of cellular stresses (reviewed by Levine, 1997) like DNA damage, transcription inhibition, depletion of nucleotide pools, oncogene expression and heat shock (Ljungman, 2000; Pluquet and Hainaut, 2001). A recent paper by Takaoka et al. (2003) demonstrated that IFN treatment of the cells induced p53 gene expression and p53-mediated apoptosis, which translated in antiviral protection. The antiviral activity of this tumor suppressor is reinforced by the fact that it is frequently targeted

*Correspondence: C Rivas; E-mail: mdcrivas@farm.ucm.es Received 5 October 2004; revised 13 December 2004; accepted 29 December 2004; published online 14 February 2005 by viral proteins. In this sense, it is known that the products of some viruses such as papillomavirus E6 protein, simian virus 40 large $\mathrm{T}$ antigen, hepatitis B X antigen, adenovirus 5 and $12 \mathrm{E} 1 \mathrm{~b}$ protein, adenovirus E4orf, Epstein-Barr virus EBNA-5 or the human cytomegalovirus IE84 protein, all target p53 (Lane and Crawford, 1979; Linzer and Levine, 1979; Kao et al., 1990; Scheffner et al., 1992; Feitelson et al., 1993; Szekely et al., 1993; Speir et al., 1994; Dobner et al., 1996).

Since accessibility of the tumor to a therapeutic agent is a major limitation of a successful antitumor therapy, oncolytic viruses provide an attractive tool for cancer treatment due to their ability to replicate selectively within the tumor and to kill neighboring cancer cells by lysis and after secondary infections (Kirn et al., 2001). One of the oncolytic viruses that is currently developed as a vector for tumor gene therapy is vesicular stomatitis virus (VSV). VSV is a negative-stranded RNA virus, prototypic member of the Rhabdoviridae family, and has several advantages as a vector for tumor destruction. The virus is extremely sensitive to the antiviral action of IFNs (Wagner and Rose, 1996), exhibits potent oncolytic activity both in vitro and in vivo (Balachandran and Barber, 2000; Stojdl et al., 2000), replicates rapidly to high titers in virtually all transformed cells, has no transforming potential, is nonhazardous to humans and has been extensively characterized immunologically (Balachandran et al., 2001). Besides, the malleability of the virus genome makes VSV an attractive vector for the delivery of proapoptotic and immunostimulatory genes into the tumor, thus potentially enhancing its oncolytic activity (Giedlin et al., 2003).

Cancer resistance can be enhanced by a genetic modification, as shown with 'super p53' mice carrying supernumerary copies of the p53 gene in the form of large genomic transgenes (Garcia-Cao et al., 2002). We have taken advantage of this experimental system to further challenge the proposed antiviral role of p53. In particular, the antiviral role of $\mathrm{p} 53$ has been so far demonstrated by comparing the response of wild-type (WT) and p53-null cells (Takaoka et al., 2003). This comparison, although highly suggestive, is nonetheless limited by the fact that the absence of p53 is likely to have large pleiotropic effects that may affect indirectly 
the antiviral response. To unequivocally test the implication of p53 in antiviral defence, without the above-mentioned confounding effects, we have compared the behavior of WT and 'super p53' cells in response to viral infection.

Primary MEFs derived from p53-/-, WT or 'super p53' mice were infected with 10 plaque-forming units $(\mathrm{PFU}) /$ cell of VSV and virus titers in supernatants, after all cells died as a result of the infection, were determined. The virus yield in WT-infected cells was around 10-fold higher than in 'super p53' MEFs and 10fold lower than in p53-/- MEFs (Figure 1a). The magnitude of the increase in the amount of virus produced from p53-/- MEFs compared to control WT MEFs was similar to that reported by Takaoka et al. (2003). The decrease in virus yield in the 'super p53'
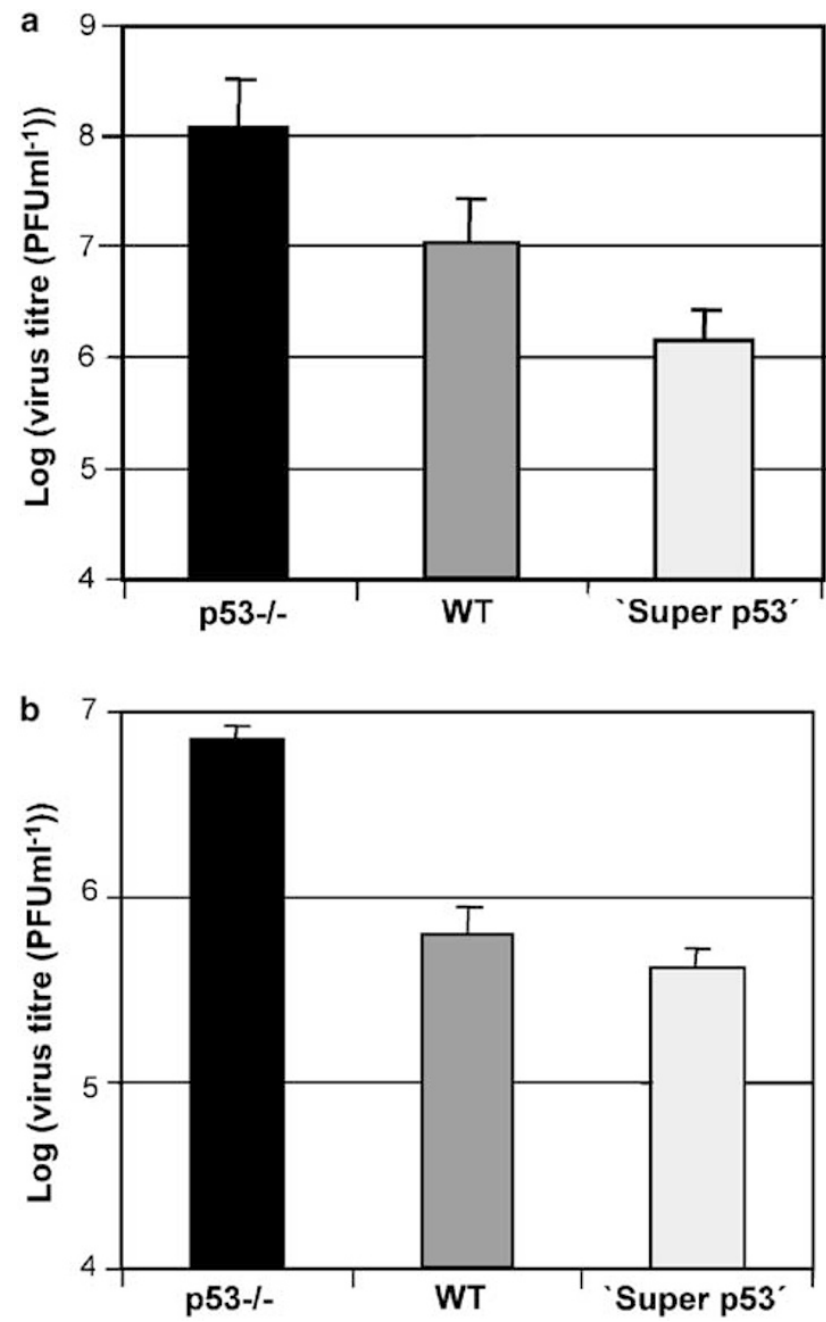

Figure 1 Correlation between p53 levels and the extent of VSV replication. Virus titers of the supernatant of the p53-/-, WT and super p53 MEFs after the viability of all the cells was lost after VSV (a) or vaccinia virus (b) infection (10 MOI) were determined by plaque assay formation. Indicated values are means of triplicate experiments. The standard deviation of the measurements was within $15 \%$ in each assay. The results for each assay were separately conducted with at least three independent clones of MEFs derived from the different mice mice-derived MEFs indicated that 'super p53'-derived fibroblasts are significantly less permissive to VSV infection. In order to investigate if the introduction of the transgene also contributes to restrict the replication of a different viral agent, we carried out similar experiments using instead a DNA virus. As shown in Figure 1b, virus yields obtained after vaccinia virus (10 PFU/cell) infection of WT MEFs was slightly higher than that obtained in transgenic MEFs and clearly lower than in p53-/- cells. However, the differences found between WT and 'super p53' MEFs were not statistically significant, indicating that the levels of p53 protein did not affect vaccinia virus infection in cultured cells. We interpret that the increased replication of vaccinia virus in p53-/ - cells is an indirect consequence of the absence of p53. In contrast, the results obtained with VSV unequivocally implicate p53 as a direct regulator of the antiviral response elicited by this virus.

The tumor suppressor p53 has the ability to trigger proliferation arrest and cell death upon the occurrence of a variety of stresses such as DNA damage, oncogenic stress or viral infection. The viral agent VSV also triggers apoptosis of the infected cells (Kopecky et al., 2001; Gadaleta et al., 2002; Sur et al., 2003). In order to determine if apoptosis induced by VSV infection in MEFs derived from the transgenic mice correlated with lower viral yields in these cells, MEFs from WT, p53-/or 'super p53' mice were infected with $1 \mathrm{PFU} /$ cell of VSV, and $24 \mathrm{~h}$ after infection, cells were processed to determine apoptosis induction. Analysis of the cells derived from the transgenic mice revealed that $89 \%$ of the cells were apoptotic. In contrast, only about $55 \%$ of the WT MEFs and $32 \%$ of the MEFs derived from the p53-/- mice infected under the same conditions were apoptotic (Figure 2a). In addition, the apoptosis induced by VSV infection correlated with the activation of the proapoptotic p53 target gene Puma (Figure 2b). The higher apoptotic level observed in WT MEFs in comparison with p53-/- cells was similar to previous findings (Takaoka et al., 2003). These results indicate that 'super p53' mice are more sensitive to apoptosis induced by VSV infection. In addition, these observations point to apoptosis as the mechanism responsible for the decreased virus replication in cells derived from transgenic mice. In contrast, analysis of the MEFs infected with vaccinia virus revealed no statistically significant differences in the levels of apoptosis induction among the different p53 genotypes (data not shown). This is probably related to induction of vaccinia virus genes known to counteract in vitro the host response at both extracellular and intracellular levels (reviewed by Smith et al., 1997), inhibiting the induction of apoptosis of the infected fibroblasts ( $\mathrm{Li}$ and $\mathrm{Beg}$, 2000).

The results obtained with MEFs (Figures 1 and 2) predict that 'super p53' mice should be more resistant to VSV infection compared to WT mice. In order to examine in vivo the higher resistance to VSV infection of the 'super p53' mice, WT or transgenic mice were intranasally inoculated with VSV $\left(1 \times 10^{4} \mathrm{PFU}\right)$ and the survival of these mice was monitored daily. Statistical 

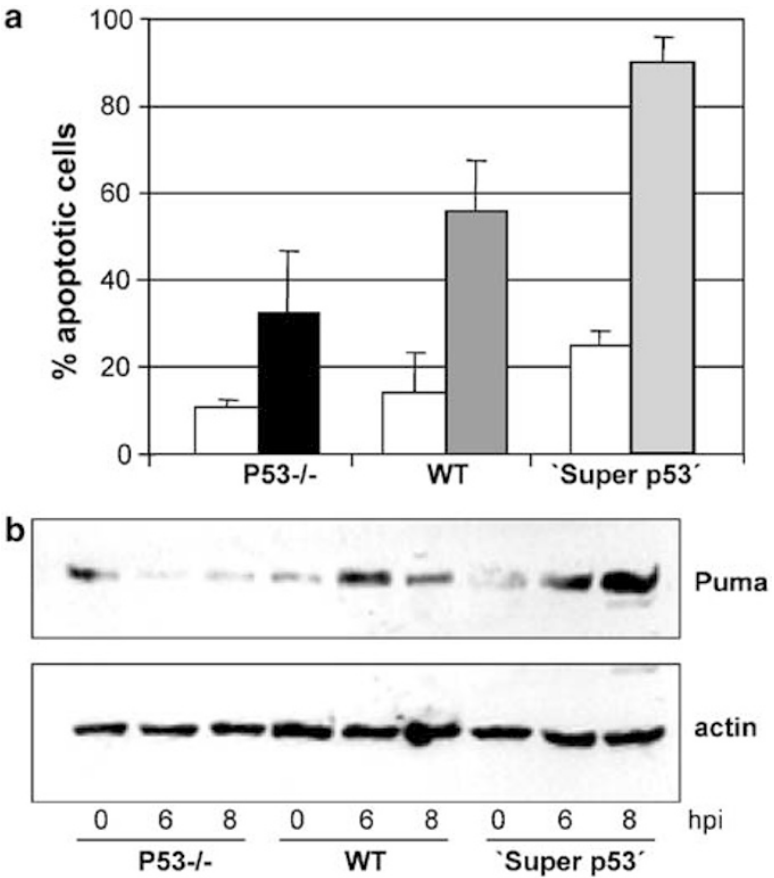

Figure 2 p53-dependent apoptosis in VSV-infected cells. (a) Correlation between p53 levels and percentage of apoptotic cells in VSV-infected cells. p53-/-, WT and super p53 MEFs were infected with VSV (1 MOI ), and $24 \mathrm{~h}$ after infection, the apoptotic response was analysed by propidium iodide staining and flow cytometry and was measured as fractions with less than G1 DNA content. Percentage of apoptosis in uninfected cells is also shown (white bars). Results shown represent the average of at least three independent experiments $(+/-$ s.d.). (b) Activation of proapoptotic p53 target gene Puma by VSV infection. p53-/-, WT and super p53 MEFs were infected with VSV (1 MOI) and cell lysates were analysed by immunoblotting with specific antibodies against Puma (Abcam Ltd, Cambridge, UK) and actin (MP Biomedicals, Aurora, $\mathrm{OH}, \mathrm{USA})$

analysis of survival rate comparison between groups was performed using the nonparametric Mann-Whitney test. We observed a slight delay in the time of death of the 'super p53' mice compared with WT animals. However, as shown in Figure 3a, all mice succumbed to VSV infection within 7 days after inoculation and the results did not show statistical differences. This result is in discordance to the significant differences in the sensitivity to VSV infection found between WT and p53-/- mice reported by Takaoka et al. (2003). These differences may be related with the intravenous route of infection used in the former studies and suggest that the route of inoculation might be critical for the antiviral action of $\mathrm{p} 53$. In addition, to further examine the susceptibility of 'super p53' mice to vaccinia virus, WT or transgenic mice were intranasally inoculated with vaccinia virus $\left(5 \times 10^{6} \mathrm{PFU}\right)$ and the weight loss was calculated at different times after infection. MannWhitney test of the percentage of weight loss at 1 week after infection revealed statistically significant differences between both groups $(P=0.049)$ (Figure 3b). Vaccinia virus infects a broad range of host cells in

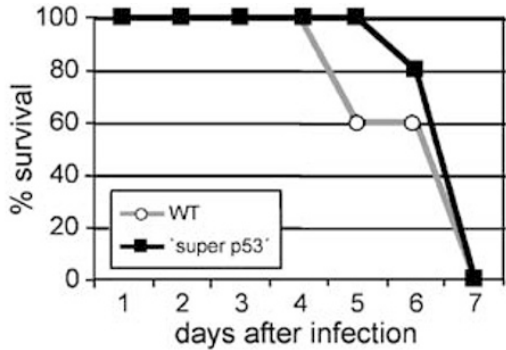

b

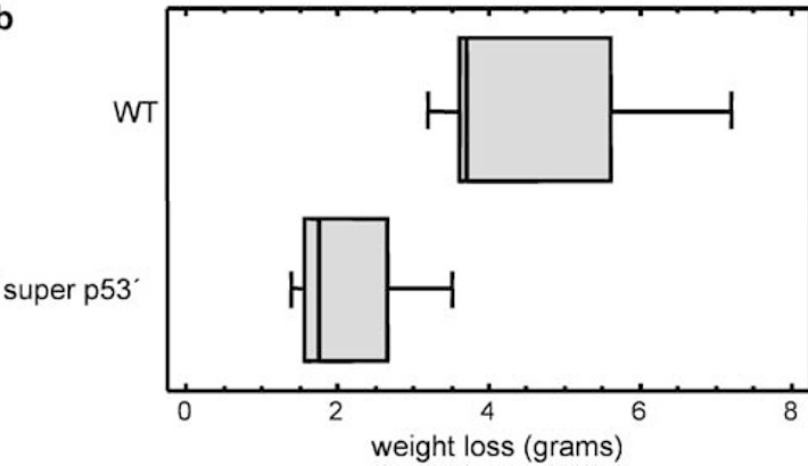

Figure 3 Susceptibility of 'super p53' mice to viral infection. (a) Survival rate of WT and 'super p53' mice after VSV infection. Five WT C57BL/6 and five 'super p53' mice of the same genetic background C57BL/6 were intranasally infected with $10^{4} \mathrm{PFU}$ VSV in $20 \mu \mathrm{l}$ at the age of 3 months and mice were daily monitored for survival. Comparison between both groups of mice of the survival rate was performed using the Mann-Whitney U test. (b), Analysis of the percentage of weight loss at one week after vaccinia virus infection. Six WT and six 'super p53' mice were intranasally infected with $5 \times 10^{6} \mathrm{PFU}$ vaccinia virus in $20 \mu \mathrm{l}$ at the age of 3 months and the weight of these mice was monitored daily. Comparison between both groups of mice was performed using the Mann-Whitney $U$-test and represented by a box-and-whisker plot. The results obtained were statistically significant different $(P=0.049)$

culture, and usually causes their lysis. Although infection of fibroblasts with vaccinia virus in vitro does not induce apoptosis, this virus can trigger an apoptotic response after infection in vivo and in macrophages (Gonzalo et al., 1994; Humlova et al., 2002). These differences might explain the variations observed between the in vivo and in vitro experiments after vaccinia virus infection.

Overall, our results reveal that increased levels of p53 might affect the resistance of the cells to be infected by specific viral agents. These results have relevance on the potential clinical utility of p53. In particular, the use of oncolytic viral agents expressing p53 might be counterproductive because it could decrease viral infection and spreading through the tumoral cells. The evaluation of the viral vector to be used for gene therapy in which normal p53 is introduced back into tumour cells has to be carefully considered.

\section{Acknowledgements}

We thank Victoria Jimenez for technical assistance on culture cells and viruses. We also thank Maribel Muñoz for great 
animal assistance. Work at the laboratory of M Serrano has been funded by the Spanish Ministry of Science and Technology (SAF2002-03402), and by the European Union (QLRT-2000-02084, QLRT-2000-00616 and INTACT).

\section{References}

Balachandran S and Barber GN. (2000). IUBMB Life, 50, 135-138.

Balachandran S, Porosnicu M and Barber GN. (2001). J. Virol., 75, 3474-3479.

Clemens MJ. (2003). J. Interferon Cytokine Res., 23, 277-292.

Dobner T, Horikoshi N, Rubenwolf S and Shenk T. (1996). Science, 272, 1470-1473.

Feitelson MA, Zhu M, Duan LX and London WT. (1993). Oncogene, 8, 1109-1117.

Gadaleta P, Vacotto M and Coulombie F. (2002). Virus Res., 86, 87-92.

Garcia-Cao I, Garcia-Cao M, Martin-Caballero M, Criado LM, Klatt P, Flores JM, Weill JC, Blasco MA and Serrano M. (2002). EMBO J., 21, 6225-6235.

Giedlin MA, Cook DN and Dubensky Jr TW. (2003). Cancer Cell, 4, 241-243.

Gonzalo JA, Gonzalez-Garcia A, Kalland T, Hedlung G, Martinez C and Kroemer G. (1994). Eur. J. Immunol., 24, 48-52.

Humlova Z, Vokurka M, Esteban M and Melkova ZJ. (2002). J. Gen. Virol., 83, 2821-2832.

Kao CC, Yew PR and Berk AJ. (1990). Virology, 179, 806-814.

Kirn D, Martuza RL and Zwiebel J. (2001). Nat. Med., 7, 781-787.

Kopecky SA, Willingham MC and Lyles DS. (2001). J. Virol., 75, 12169-12181.
C Rivas laboratory work was supported by a grant from Ministerio de Ciencia y Tecnologia de España (BIO200202417). C Rivas is an investigator of the Ramon y Cajal Programme.

Lane DP and Crawford L. (1979). Nature, 278, 261-263.

Levine AJ. (1997). Cell, 88, 323-331.

Li M and Beg AA. (2000). J. Virol., 74, 7470-7477.

Linzer DI and Levine AJ. (1979). Cell, 17, 43-52.

Ljungman M. (2000). Neoplasia, 2, 208-225.

Pluquet O and Hainaut P. (2001). Cancer Lett., 174, 1-15.

Samuel CE. (2001). Clin. Microbiol. Rev., 14, 778-809.

Scheffner M, Takahashi T, Huibregtse JM, Minna JD and Howley PM. (1992). J. Virol., 66, 5100-5105.

Smith GL, Symons JA, Khanna A, Vaderplasschen A and Alcami A. (1997). Immunol. Rev., 159, 137-154.

Speir E, Modali R, Huang ES, Leon MB, Shawl F, Finkel T and Epsteins E. (1994). Science, 265, 391-394.

Stojdl DF, Lichty B, Knowles S, Marius R, Atkins H, Sonenberg N and Bell JC. (2000). Nat. Med., 6, $821-825$

Sur JH, Allende R and Doster AR. (2003). Vet. Pathol., 40, $512-520$.

Szekely L, Selivanova G, Magnusson KP, Klein G and Wiman KG. (1993). Proc. Natl. Acad. Sci. USA, 90, 5455-5459.

Takaoka A, Hayakawa S, Yanai H, Stoiber D, Negishi H, Kikuchi H, Sasaki S, Imai K, Shibue T, Honda K and Taniguchi T. (2003). Nature, 424, 516-523.

Wagner RR and Rose JK. (1996). Fields Virology, 3rd edn. Fields BN, Knipe DM and Howley PM (ed). LippincottRaven Publishers: Philadelphia, PA. 